\title{
Augmenting Forests to Meet Odd Diameter Requirements *
}

\author{
Toshimasa Ishii ${ }^{a}{ }^{*}$, Shigeyuki Yamamoto ${ }^{b}$, Hiroshi Nagamochi ${ }^{c}$ \\ ${ }^{a}$ Department of Information and Computer Sciences, \\ Toyohashi University of Technology, \\ Aichi 441-8580, Japan \\ ${ }^{\mathrm{b}}$ I FOR COM Co.,Ltd. Kanagawa 220-0207, Japan \\ ${ }^{\mathrm{c}}$ Department of Applied Mathematics and Physics, \\ Graduate School of Informatics, \\ Kyoto University, \\ Kyoto 606-8501, Japan
}

\begin{abstract}
Given a graph $G=(V, E)$ and an integer $D \geq 1$, we consider the problem of augmenting $G$ by the smallest number of new edges so that the diameter becomes at most $D$. It is known that no constant approximation algorithms to this problem with an arbitrary graph $G$ can be obtained unless $P=N P$. For a forest $G$ and an odd $D \geq 3$, it was open whether the problem is approximable within a constant factor. In this paper, we give the first constant factor approximation algorithm to the problem with a forest $G$ and an odd $D$; our algorithm delivers an 8-approximate solution in $O\left(|V|^{3}\right)$ time. We also show that a 4 -approximate solution to the problem with a forest $G$ and an odd $D$ can be obtained in linear time if the augmented graph is additionally required to be biconnected.
\end{abstract}

Key words: undirected graph, graph augmentation problem, diameter, forest, approximation algorithm

\footnotetext{
ॠ An extended abstract of this paper will be presented at 14th International Symp. on Algorithms and Computation (ISAAC'2003), Kyoto, December 2003.

* Corresponding author.

Email addresses: ishii@ics.tut.ac.jp (Toshimasa Ishii), nag@amp.i.kyoto-u.ac.jp (Hiroshi Nagamochi).
} 


\section{Introduction}

In communication networks, some transfer delay occurs when we send a message from one node to another node. The least number of links through which the message has to be transmitted is considered as one measurement of such a transfer delay. Therefore, it is desirable that a network has a small diameter, which is defined as the maximum distance between every two nodes in the network. In $[1,6,7,9,13,15]$, the problems of constructing a graph with a small diameter by adding new edges to an initial graph have been studied, as one of the network design problems such as airplane flights scheduling [7].

Given an undirected graph $G=(V, E)$ and a nonnegative integer $D$, the augmentation problem with diameter requirements (for short, $A P D$ ) is to augment $G$ by adding the smallest number of new edges that reduces the diameter to at most $D$. Note that the case of $D=1$ is trivial, because only the complete graph can have diameter one. In general, Schoone et al. [15] have shown that APD is NP-hard for any fixed $D \geq 3$. Moreover, it has been shown that there is no constant approximation algorithm to $\mathrm{APD}$ unless $\mathrm{P}=\mathrm{NP}$, by a reduction from DOMINATING SET due to Li et al. [13] for $D \geq 4$, and by a reduction from SET COVER due to Dodis and Khanna [8] for $D \in\{2,3\}$. The same results have been shown by Chepoi and Vaxes [6]. Let $O P T_{A}(G, D)$ denote the optimal value to APD with a graph $G$ and an integer $D$. Alon et al. [1] have shown that $\operatorname{OPT}_{A}(G, 2)=n-\Delta-1$ and $\operatorname{OPT}_{A}(G, 3) \geq n-O\left(\Delta^{3}\right)$ hold for any graph $G$ with the maximum degree $\Delta$ and a sufficiently large number $n=|V|$ of vertices and that $O P T_{A}(G, D) \leq n /\lfloor D / 2\rfloor$ holds for any connected graph $G$. Also for APD with some restricted classes of graphs, several problems have been studied. Erdős et al. [9] have investigated upper and lower bounds on the optimal value to APD in the case where a given graph and an augmented graph are restricted to be triangle-free. Alon et al. [1] have proved that $O P T_{A}\left(C_{n}, D\right)=n /(2\lfloor D / 2\rfloor-1)-O(1)$ holds for any cycle $C_{n}$ of $n$ vertices. Recently Chepoi and Vaxes [6] have presented a 2-approximation algorithm to APD with a forest and an even integer $D$. They have also proved that their algorithm can be applied to a wider class of graphs $G$ satisfying the following conditions (i) and (ii). (i) $G$ is a Helly graph (see [2] for the definition). (ii) There exists a polynomial time algorithm to the $k$-DOMINATING SET with $G$, the problem of finding a smallest set $X$ of vertices such that the distance from each vertex to some vertex in $X$ is at most $k$. Forests and dually chordal graphs (see [3] for the definition) are included in such a class of graphs. However, it was left open whether APD with an odd diameter is approximable by a constant factor or not, even if $G$ is a forest, while it is also left open whether APD with a forest is NP-hard or not.

As a related problem, we consider APD with an additional requirement that the resulting augmented graph is a biconnected graph, i.e., it has at least two 
vertex-disjoint paths between every two vertices. This problem is called the biconnectivity augmentation problem with diameter requirements (for short, $B A P D)$. In communication networks, graph connectivity can be considered as a fundamental measure of its robustness. Eswaran and Tarjan [10] have shown that the problem of augmenting an initial graph up to biconnectivity can be solved in linear time. For graph connectivities augmentation problems, many problems and algorithms have been studied (see [11,14] for surveys). Chepoi and Vaxes [6] have proved that BAPD is NP-hard even if $G$ is a tree.

Definition 1 Let us call a solution $(a, b)$-approximate solution if the number of edges in the solution is at most $b$ surplus edges over a times the optimal, and an algorithm that delivers such a solution an ( $a, b)$-approximation algorithm.

Chepoi and Vaxes have also given a 3-approximation algorithm for an even integer $D[6]$, and a $(7,3)$-approximation (resp., $(9,4)$-approximation) algorithm for an odd $D \geq 5$ (resp., $D=3$ ) [5], in the case where $G$ is a forest.

In this paper, we consider designing an approximation algorithm to APD and BAPD, in the case where an initial graph $G$ is a forest and $D$ is an odd integer. We partly follow Chepoi and Vaxes' approaches [6] to obtain a 2-approximate solution to APD with forest $G$ and the even integer $D+1$, which is a relaxation of the original problem instance $(G, D)$. Unfortunately, it is not difficult to see that $\operatorname{OPT}_{A}(G, D) / O P T_{A}(G, D+1)$ cannot be bounded from above by any constant even in the case of trees. We establish a new lower bound on the optimal value to APD with an odd $D$. With the 2-approximate solution to the even $D+1$ and the new lower bound on $\operatorname{OPT}_{A}(G, D)$, we prove that an 8approximate solution to APD can be constructed in $O\left(|V|^{3}\right)$ time. For BAPD, we propose an $O(|V|)$ time (4,2)-approximation (resp., (6,3)-approximation) algorithm for an odd $D \geq 5$ (resp., $D=3$ ).

The paper is organized as follows. In Section 2, we state our main results that APD with a forest and an odd $D$ is 8-approximable and BAPD with a forest and an odd $D \geq 5$ (resp., $D=3$ ) is (4,2)-approximable (resp., (6,3)approximable), after introducing some basic notation. In Section 3, we propose an 8-approximation algorithm, named $\operatorname{ODD}-\operatorname{APD}(G, D)$, to APD with a forest $G$ and an odd $D$, after reviewing Chepoi and Vaxes' algorithm [6] to APD with a forest and an even $D$. In Section 4 , we give an approximation algorithm to BAPD with a forest $G$ and an odd $D$. In Section 5, we give concluding remarks. 


\section{Preliminaries}

Let $G=(V, E)$ stand for an undirected simple graph with a set $V$ of vertices and a set $E$ of edges. An edge with end vertices $u$ and $v$ is denoted by $(u, v)$. We denote $|V|$ by $n$ and $|E|$ by $m$. A singleton set $\{x\}$ may be simply written as $x$, and " $\subset$ " implies proper inclusion while " $\subseteq$ " means " $\subset$ " or " $=$ ". In $G=(V, E)$, its vertex set $V$ and edge set $E$ may be denoted by $V(G)$ and $E(G)$, respectively. For a subset $V^{\prime} \subseteq V$ in $G, G\left[V^{\prime}\right]$ denotes the subgraph induced by $V^{\prime}$. For an edge set $E^{\prime}$, we denote by $V\left[E^{\prime}\right]$ the set of all end vertices of edges in $E^{\prime}$. For a vertex set $X \subset V$ in a graph $G$, we denote by $N_{G}(X)$ the set of vertices in $V-X$ adjacent to some vertex $v \in X$.

The length of a path $P$ is defined by the number of edges in $P$ and is denoted as $|P|$ (i.e., $|P|=|E(P)|)$. For two vertices $u, v \in V$ in $G=(V, E)$, the distance between $u$ and $v$ is defined as the length of a path between $u$ and $v$ with the shortest length, and it is denoted by $d_{G}(u, v)$. The diameter of a graph $G$, denoted by $\operatorname{diam}(G)$, is defined as the maximum among distances between all pairs of vertices in $G$. For a vertex $u \in V$ in a graph $G$ and an integer $k$, let $N_{G}^{k}(u)$ denote the set of vertices $v$ with $d_{G}(u, v)=k$. A set $B_{G}(u, k)=\bigcup_{k^{\prime} \leq k} N_{G}^{k^{\prime}}(u)$ of vertices is called the ball centered at $u$ of radius $k$. $B_{G}(u, k)$ may be simply called a $k$-ball (with a center $u$ ). For a subset $V^{\prime} \subseteq V$ of vertices and a family $\mathcal{B}$ of balls, we say that $\mathcal{B}$ covers $V^{\prime}$ if every vertex in $V^{\prime}$ is contained in some ball in $\mathcal{B}$.

A forest is a graph with no cycle. For a forest $G=(V, E)$, a vertex $v \in V$ with degree 1 or 0 is called a leaf, and we denote the set of all leaves in $G$ by $L(G)$. For a set $X$ of vertices in a forest $G$, two vertices $x_{1}$ and $x_{2}$ in $V$ are called adjacent with respect to $X$ if the path between $x_{1}$ and $x_{2}$ does not contain any vertex in $X-\left\{x_{1}, x_{2}\right\}$ (note that a path between two vertices is uniquely determined in a forest). A graph $G=(V, E)$ with $|V| \geq k+1$ is called $k$-vertex-connected, if the deletion of any vertex set $X$ with $|X| \leq k-1$ leaves a connected graph. The vertex-connectivity of $G$, denoted by $\kappa(G)$, is defined as the largest integer $k$ for which $G$ is $k$-vertex-connected.

In this paper, we consider the following two problems.

Problem 2 Augmentation Problem with Diameter Requirements (APD) Input: A graph $G=(V, E)$ and a nonnegative integer $D$.

Output: $A$ set $E^{*}$ of edges with the minimum cardinality such that $\operatorname{diam}\left(G^{*}\right)$ $\leq D$ holds for $G^{*}=\left(V, E \cup E^{*}\right)$.

Problem 3 Biconnectivity Augmentation Problem with Diameter Requirements $(B A P D)$

Input: $A$ graph $G=(V, E)$ with $|V| \geq 3$ and a nonnegative integer $D$.

Output: A set $E^{*}$ of edges with the minimum cardinality such that $\operatorname{diam}\left(G^{*}\right)$ 
$\leq D$ and $\kappa\left(G^{*}\right) \geq 2$ hold for $G^{*}=\left(V, E \cup E^{*}\right)$.

Let $\operatorname{OPT}_{A}(G, D)$ and $O P T_{B}(G, D)$ denote the optimal value to APD and BAPD with $G$ and an integer $D$, respectively. For these problems, we show the following two theorems.

Theorem 4 If $G$ is a forest and $D$ is an odd integer, then an 8-approximate feasible solution to APD can be found in $O\left(n^{3}\right)$ time.

Theorem 5 Let $G$ be a forest. Then a $(4,2)$-approximate $((6,3)$-approximate) feasible solution to BAPD can be found in $O(n)$ time if $D$ is an odd $\geq 5$ (resp., $D=3$ holds).

\section{APD with a forest}

In this section, let $G=(V, E)$ be a forest and $D=2 R+1$ be an odd integer with $R \geq 1$. We show that APD is 8-approximable in $O\left(n^{3}\right)$ time in the case where $G$ is a forest and $D$ is an odd integer.

Let $P_{u, v}$ denote a path between two vertices $u$ and $v$ in $G$ (note that $P_{u, v}$ is uniquely determined if $G$ is a forest). We first find a 2-approximate solution $E_{1}$ to $A P D$ with the forest $G$ and the even $D^{\prime}=D+1$ by Chepoi and Vaxes' algorithm [6]. Note that $\left|E_{1}\right| / 2$ is a lower bound on $\operatorname{OPT}_{A}(G, D)$ since we have $\operatorname{OPT}_{A}(G, D) \geq O \operatorname{OPT}_{A}(G, D+1) \geq\left|E_{1}\right| / 2$. We then construct an 8approximate solution to APD with $G$ and $D$ based on the edge set $E_{1}$. In this section, we first review Chepoi and Vaxes' algorithm [6] in Section 3.1, analyze properties of solutions by their algorithm, derive another lower bound on $\operatorname{OPT}_{A}(G, D)$, and finally propose an 8-approximation algorithm based on these analyses in Section 3.2.

\subsection{Even diameters}

The following algorithm EVEN-APD is a 2-approximation algorithm to APD with a forest $G$ and an even $D^{\prime}$ by Chepoi and Vaxes [6].

\section{Algorithm EVEN-APD $\left(G, D^{\prime}\right)$}

Input: A forest $G=(V, E)$ and an even integer $D^{\prime} \geq 2$.

Output: A new edge set $E_{1}$ with $\operatorname{diam}\left(\left(V, E \cup E_{1}\right)\right) \leq D^{\prime}$ and $\left|E_{1}\right| \leq$ $2 O P T_{A}\left(G, D^{\prime}\right)$. 
Step 1: Let $R=\frac{D^{\prime}-2}{2}$. Choose a center $c^{*} \in V$ for the $(R+1)$-ball and a set $C_{1}$ of centers for $R$-balls so that the family of these $\left|C_{1}\right|+1$ balls covers $V$ in $G$ and the number $\left|C_{1}\right|+1$ of centers is minimized. Halt after outputting the set $E_{1}=\left\{\left(c^{*}, c\right) \mid c \in C_{1}\right\}$ of new edges.

For the completeness of the paper, we give a sketch of the proof for the correctness of algorithm EVEN-APD.

Theorem 6 [6] The edge set $E_{1}$ obtained by algorithm EVEN-APD $\left(G, D^{\prime}\right)$ satisfies $\left|E_{1}\right| \leq 2 O P T_{A}\left(G, D^{\prime}\right)$, and can be found in $O(n m)$ time.

Proof sketch: Let $E^{*}$ be an optimal solution to APD with $G$ and $D^{\prime}=2 R+2$, and $W=V\left[E^{*}\right]$. Let $X=V-\left(\bigcup_{w \in W} B_{G}(w, R)\right) ; X$ is the set of vertices which are not contained in $B_{G}(w, R)$ for any $w \in W$. Then we can prove from $\operatorname{diam}\left(\left(V, E \cup E^{*}\right)\right) \leq 2 R+2$ that $d_{G}(x, y) \leq 2 R+2$ holds for every two vertices $x$ and $y$ in $X$ and that there is a vertex $v^{*} \in V$ with $X \subseteq B_{G}\left(v^{*}, R+1\right)$. Moreover, the edge set $E^{\prime}=\left\{\left(v^{*}, w\right) \mid w \in W\right\}$ satisfies $\operatorname{diam}\left(\left(V, E \cup E^{\prime}\right)\right) \leq 2 R+2$ and $\left|E^{\prime}\right| \leq|W| \leq 2\left|E^{*}\right|$. From the construction of $E_{1}$, we have $\left|E_{1}\right| \leq\left|E^{\prime}\right| \leq 2\left|E^{*}\right|$ $=2 O P T_{A}\left(G, D^{\prime}\right)$.

Let $H_{1}=\left(V, E \cup E_{1}\right)$ and $C_{2}=N_{G}\left(c^{*}\right)$. The following lemma holds from the construction of $E_{1}$.

Lemma $7(i)\left|C_{1}\right|=\left|E_{1}\right| \leq 2 O P T_{A}(G, 2 R+2) \leq 2 O P T_{A}(G, 2 R+1)$ holds. (ii) The family of $R$-balls with centers in $C_{1} \cup C_{2}$ covers $V$ if $R \geq 1$.

(iii) In $H_{1}$, every vertex $v \in V$ satisfies $d_{H_{1}}\left(c^{*}, v\right) \leq R+1$.

(iv) Every two vertices $u_{1}, u_{2} \in V$ with $d_{H_{1}}\left(u_{1}, u_{2}\right)>2 R+1$ satisfy $d_{H_{1}}\left(u_{1}\right.$, $\left.u_{2}\right)=2 R+2$ and $d_{H_{1}}\left(c^{*}, u_{1}\right)=d_{H_{1}}\left(c^{*}, u_{2}\right)=R+1$; such a vertex $u_{i}$ satisfies $d_{G}\left(u_{i}, c\right)=R$ for some $c \in C_{1} \cup C_{2}$.

\subsection{Odd diameters}

In this section, we propose an algorithm, named $\operatorname{ODD}-\mathrm{APD}(G, D)$, for constructing a solution to APD with an odd diameter $D=2 R+1$. This algorithm consists of the following three steps. In the first step, we compute a center $c^{*}$, a set $C_{1}$ of centers and the set $C_{2}=N_{G}\left(c^{*}\right)$ in Lemma 7 , and augment $G$ by the new edge set $E_{1}=\left\{\left(c^{*}, v\right) \mid v \in C_{1}\right\}$. In $H_{1}=\left(V, E \cup E_{1}\right)$, there may be a vertex $u \in V$ such that $d_{H_{1}}\left(u, u^{\prime}\right)>2 R+1$ for some other vertex $u^{\prime}$. We call such a vertex $u$ distant. By Lemma 7 (iv), we see that $d_{H_{1}}\left(u, u^{\prime}\right)=2 R+2$ holds. Thus, to make the diameter at most $D=2 R+1$, it suffices to decrease by at least one the distance between those vertices in the second and third steps. In the second step, we compute a set $C_{3}$ by choosing at most $2\left|C_{1}\right|$ vertices from 

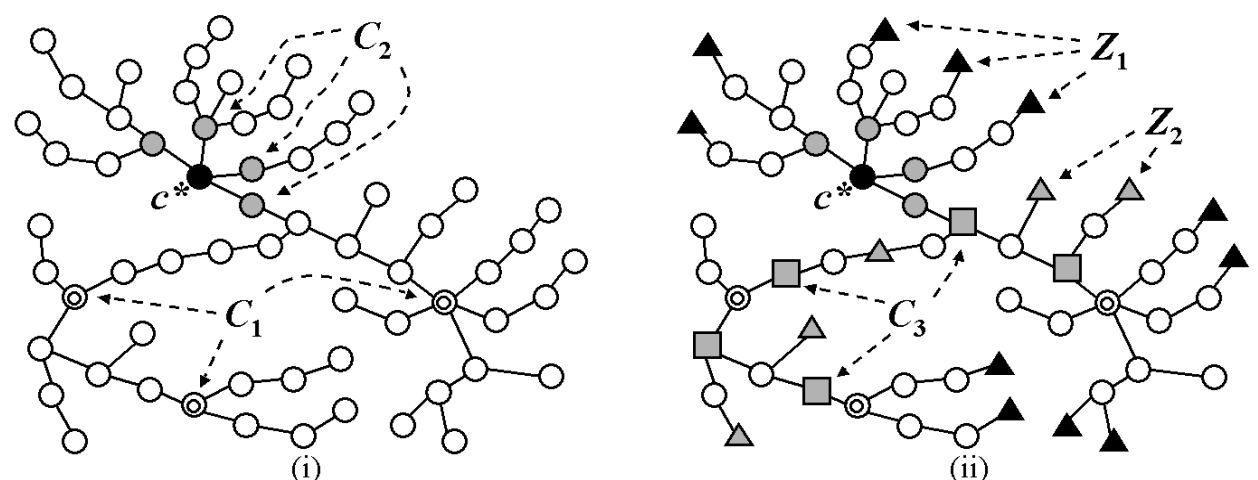

Fig. 1. (i) Illustration of a tree $G$, a vertex $c^{*}$ and sets $C_{1}$ and $C_{2}$ of vertices obtained by applying Step 1 of algorithm $\operatorname{ODD}-\operatorname{APD}(G, 7)$ to $G$, where each vertex in $C_{1}$ (resp., $C_{2}$ ) is drawn by a double circle (resp., a shaded circle). (ii) Illustration of distant vertices and a set $C_{3}$ of vertices, where each distant vertex in $Z_{1}$ (resp., $Z_{2}$ ) is drawn by a black (resp., shaded) triangle, and each vertex in $C_{3}$ is drawn by a shaded square.

$N_{G}\left(C_{1} \cup C_{2}\right)$ and augment $H_{1}$ by the new edge set $E_{2}=\left\{\left(c^{*}, v\right) \mid v \in C_{3}\right\}$, by which $H_{2}=\left(V, E \cup E_{1} \cup E_{2}\right)$ satisfies $d_{H_{2}}\left(c^{*}, v\right) \leq R$ for some distant vertex $v$ in $H_{1}$. In the third step, we augment $H_{2}$ by a new edge set $E_{3}$ with $\left|E_{3}\right| \leq 2 O P T_{A}(G, D)$ so that in $H_{3}=\left(V, E \cup E_{1} \cup E_{2} \cup E_{3}\right)$, every distant vertex $u$ in $H_{1}$ now satisfies $d_{H_{3}}\left(u, u^{\prime}\right) \leq 2 R+1$ for all vertices $u^{\prime} \in V$.

More precisely, algorithm $\operatorname{ODD}-\operatorname{APD}(G, D)$ is described as follows, where $E_{3}$ in Step 3 is constructed based on our new lower bound on $\operatorname{OPT}_{A}(G, D)$, and how to choose such $E_{3}$ will be described after verifying Step 2.

\section{Algorithm ODD-APD $(G, D)$}

Input: A forest $G=(V, E)$ and an odd integer $D \geq 3$.

Output: An set $E^{*}$ of edges with $\operatorname{diam}\left(\left(V, E \cup E^{*}\right)\right) \leq D$ and $\left|E^{*}\right| \leq$ $8 O P T_{A}(G, D)$.

Step 1: Let $R=\frac{D-1}{2}$. Compute a center $c^{*}$, a set $C_{1}$ of centers, the set $C_{2}=N_{G}\left(c^{*}\right)$ and the edge set $E_{1}$ that satisfy Lemma 7 .

Step 2: Regard each component $G^{\ell}$ of $G$ as a rooted tree by choosing its root $c^{\ell}$ as $c^{*}$ if $c^{*} \in V\left(G^{\ell}\right)$ and as an arbitrary vertex in $C_{1} \cap V\left(G^{\ell}\right)$ otherwise. For each vertex $c \in C_{1}$, let $Q_{c}=N_{G}(\{c, p\}) \cap V\left(P_{c, p}\right)$ for the nearest ancestor $p \in C_{1} \cup C_{2}$ of $c$ in the rooted tree $G^{\ell}$ containing $c$. Let $C_{3}=\cup_{c \in C_{1}} Q_{c}$, $E_{2}=\left\{\left(c^{*}, v\right) \mid v \in C_{3}\right\}$, and $H_{2}=\left(V, E \cup E_{1} \cup E_{2}\right)$.

Step 3: Compute a set $E_{3}$ of edges such that $\left|E_{3}\right| \leq 2 O P T_{A}(G, D)$ and $d_{\left(V, E \cup E_{3}\right)}(u, v) \leq 2 R+1$ holds for every two vertices $u, v \in V$ with $d_{H_{2}}(u, v)>$ 
$2 R+1$. Halt after outputting $E^{*}=E_{1} \cup E_{2} \cup E_{3}$.

We first prove the correctness of algorithm $\operatorname{ODD}-\operatorname{APD}(G, D)$ under the assumption that Step 3 works correctly, by Lemmas $8-10$. These lemmas are derived almost directly from the structure of edge sets $E_{1} \cup E_{2}$ obtained in Steps 1 and 2. After that, we will describe the procedure of Step 3 for the details and show its correctness.

Let $Z$ denote the set of all distant vertices in $H_{1}=\left(V, E \cup E_{1}\right)$ (see Fig. 1 where $D=7$ ). Let $Z_{1}$ be the set of distant vertices $u \in Z$ such that $u$ has only one vertex in $C_{1} \cup C_{2}$ that is adjacent to $u$ with respect to $C_{1} \cup C_{2}$ in $G$ (recall the definition of "adjacent with respect to a vertex set in a forest" given in Section 2). Let $Z_{2}=Z-Z_{1}$. For $Q_{c}$ obtained in Step 2, we have $\left|Q_{c}\right| \leq 2$ for each $c \in C_{1}$ and $C_{3} \subseteq \bigcup_{c \in C_{1} \cup C_{2}} N_{G}(c)$, and hence we see that the following properties hold.

Lemma 8 (i) $\left|C_{3}\right| \leq 2\left|C_{1}\right|$ holds. (ii) For two vertices $c_{i}, c_{j} \in C_{1} \cup C_{2}$ adjacent with respect to $C_{1} \cup C_{2}$, assume that the path $P_{c_{i}, c_{j}}$ satisfies $\left|P_{c_{i}, c_{j}}\right| \geq 2$. Then we have $\left|P_{c_{i}, c_{j}}\right|=2$ and $c^{*} \in V\left(P_{c_{i}, c_{j}}\right)$ if $\left\{c_{i}, c_{j}\right\} \subseteq C_{2}$ holds, and we have $V\left(P_{c_{i}, c_{j}}\right) \cap N_{G}\left(\left\{c_{i}, c_{j}\right\}\right) \subseteq C_{3}$ otherwise.

Lemma 9 Every distant vertex $u \in Z_{2}$ satisfies $d_{H_{2}}\left(u, u^{\prime}\right) \leq 2 R+1$ for any vertex $u^{\prime} \in V$.

PROOF. It suffices to show that every distant vertex $u \in Z_{2}$ satisfies $d_{H_{2}}\left(c^{*}\right.$, $u) \leq R$ by Lemma 7(iii). Let $c \in C_{1} \cup C_{2}$ be a vertex with $d_{G}(c, u)=R$ (such $c$ exists by Lemma $7($ iv) $)$. From the definition of $Z_{2}$, it is not difficult to see that $\left(V\left(P_{c, u}\right) \cap V\left(P_{c, c^{\prime}}\right)\right)-\left\{c, c^{\prime}\right\} \neq \emptyset$ holds in $G$ for some vertex $c^{\prime} \in C_{1} \cup C_{2}$. This implies that $\left|P_{c, c^{\prime}}\right| \geq 2$ and $\left\{c, c^{\prime}\right\}-C_{2} \neq \emptyset$ hold. Hence, by Lemma 8(ii), the vertex $v$ with $V\left(P_{c, c^{\prime}}\right) \cap N_{G}(c)=\{v\}$ satisfies $v \in C_{3}$. Thus $v \in V\left(P_{c, u}\right)$ and $\left(c^{*}, v\right) \in E_{2}$ indicate that $d_{\left(V, E \cup E_{2}\right)}\left(u, c^{*}\right) \leq d_{G}(u, v)+1=R$ holds.

By Lemmas 7(i) and 8(i), we have $\left|E_{1} \cup E_{2}\right| \leq 3\left|C_{1}\right| \leq 6 O P T_{A}(G, D)$. Therefore, the following lemma holds.

Lemma 10 Assume that such an edge set $E_{3}$ in Step 3 can be found. Then the edge set $E^{*}$ obtained by algorithm $O D D-A P D(G, D)$ satisfies diam $((V, E \cup$ $\left.\left.E^{*}\right)\right) \leq D$ and $\left|E^{*}\right| \leq 8 O P T_{A}(G, D)$.

In the rest of this section, we show how to find $E_{3}$ in Step 3 of algorithm $\operatorname{ODD}-\operatorname{APD}(G, D)$. For this, we establish the following lemmas. In order to specify distant vertices in $Z_{1}$, we divide $G$ into subgraphs $G_{i}$ corresponding to $c_{i} \in C_{1} \cup C_{2}$ in the following manner. Let $G_{i}$ be the component containing 
$c_{i} \in C_{1} \cup C_{2}$ in $G^{\prime}$ which is obtained from $G$ by removing the set $C_{3} \cup\left\{c^{*}\right\}$ of vertices and the set $\left\{\left(c, c^{\prime}\right) \in E \mid c, c^{\prime} \in C_{1} \cup C_{2}\right\}$ of edges (see Fig. 2(i)).

Lemma 11 (i) $V\left(G_{i}\right) \cap\left(C_{1} \cup C_{2}\right)=\left\{c_{i}\right\}$ holds.

(ii) A vertex $u \in V-\left(C_{1} \cup C_{2}\right)$ is contained in $G_{i}$ if and only if $c_{i}$ is the only vertex in $C_{1} \cup C_{2}$ that is adjacent to $u$ with respect to $C_{1} \cup C_{2}$.

(iii) Every vertex $v \in V\left(G_{i}\right)$ satisfies $d_{G}\left(c_{i}, v\right) \leq R$. Moreover, each vertex $v \in V\left(G_{i}\right)$ with $d_{G}\left(c_{i}, v\right)=R$ is a leaf also in $G$.

(iv) Every distant vertex $v \in Z_{1}$ is contained in some $G_{i}$, and it is a leaf in $G$ with $d_{G}\left(c_{i}, v\right)=R$.

PROOF. (i) Let $c_{i}$ and $c_{j}$ be two vertices in $C_{1} \cup C_{2}$ adjacent to each other with respect to $C_{1} \cup C_{2}$. If $d_{G}\left(c_{i}, c_{j}\right)=1$ (resp., $d_{G}\left(c_{i}, c_{j}\right) \geq 2$ ) holds, then $c_{i}$ and $c_{j}$ are contained in distinct components in $G^{\prime}$ by $E\left(G^{\prime}\right) \cap\left\{\left(c_{i}, c_{j}\right) \in E\right.$ $\left.c_{i}, c_{j} \in C_{1} \cup C_{2}\right\}=\emptyset$ (resp., by Lemma 8 (ii) and $\left(C_{3} \cup\left\{c^{*}\right\}\right) \cap V\left(G^{\prime}\right)=\emptyset$ ).

(ii) It is not difficult to see that the property follows from the construction of $G_{i}$.

(iii) By (ii), $d_{G}\left(c_{i}, v\right) \leq R$ holds since $v$ must be contained in the $R$-ball with the center $c_{i}$. Moreover, we see that if $d_{G}\left(c_{i}, v\right)=R$ holds, then $v$ is a leaf in $G$.

(iv) Assume that $v$ is a distant vertex in $Z_{1}$. From (ii), the definition of $Z_{1}$, and the construction of $G_{i}, v$ is contained in some $G_{i}$ having $c_{i} \in C_{1} \cup C_{2}$. Lemma 7 (iv) and the above statement (iii) say that $d_{G}\left(c_{i}, v\right)=R$ holds and $v$ is a leaf in $G$.

Since each distant vertex in $Z_{1}$ is a leaf in $G$ by Lemma 11 (iv), we call a vertex $v \in Z_{1}$ a distant leaf. Let $G_{i}^{j}, j=1,2, \ldots, g_{i}$ be the component in $G_{i}-c_{i}$ such that $V\left(G_{i}^{j}\right)$ contains a distant leaf, where $g_{i}$ denotes the number of such subgraphs $G_{i}^{j}$ in $G_{i}$.

Lemma 12 Let $G_{i}^{j}$ be a component in $G_{i}-c_{i}$ such that $V\left(G_{i}^{j}\right)$ contains a distant leaf, and $v \in V\left(G_{i}^{j}\right)$ be a distant leaf in $G_{i}$. For any vertex $x \in V-$ $V\left(G_{i}^{j}\right)-\left\{c_{i}\right\}$, we have $d_{G}(v, x)>R$.

PROOF. From the definition of $G_{i}^{j}$, each vertex $x \in V\left(G_{i}\right)-V\left(G_{i}^{j}\right)-\left\{c_{i}\right\}$ satisfies $d_{G}(v, x)>R$. Assume that $x \in V-V\left(G_{i}\right)$ holds. Lemma 11(ii) implies that the path $P_{x, v}$ always contains the vertex $c_{i}$. Since $d_{G}\left(c_{i}, v\right)=R$ holds by Lemma 11(iv), we have $d_{G}(v, x)>R$. 
Let $\mathcal{G}=\left\{G_{1}, G_{2}, \ldots, G_{t}\right\}$ be the family of subgraphs $G_{i}$ which have distant leaves, where $g_{1} \geq g_{2} \geq \cdots \geq g_{t}$ holds. Let $V\left(G_{i}^{j}\right) \cap N_{G}\left(c_{i}\right)=\left\{a_{i}^{j}\right\}$ for each $G_{i} \in \mathcal{G}$ (note that $\left|V\left(G_{i}^{j}\right) \cap N_{G}\left(c_{i}\right)\right|=1$ holds from the construction of $\left.G_{i}^{j}\right)$. We here establish a new lower bound on $O P T_{A}(G, 2 R+1)$. Let $E^{\prime}$ be an arbitrary feasible solution to APD. If no edge in $E^{\prime}$ is incident to any vertex in $V\left(G_{i}^{k}\right) \cup V\left(G_{j}^{\ell}\right)$ for some $G_{i}^{k}$ and $G_{j}^{\ell}, i \neq j$, then $\left(c_{i}, c_{j}\right) \in E$ or $\left(c_{i}, c_{j}\right) \in E^{\prime}$ must hold. It follows since otherwise Lemma 12 implies that $d_{\left(V, E \cup E^{\prime}\right)}(x, y)>D$ would hold for two distant leaves $x \in V\left(G_{i}^{k}\right)$ and $y \in V\left(G_{j}^{\ell}\right)$. Hence for the family $\mathcal{G}^{\prime}$ of $G_{i}$ such that some $G_{i}^{k}$ has no edge in $E^{\prime}$ incident to it, the set of the corresponding centers $c_{i}$ induces a complete graph in $\left(V, E \cup E^{\prime}\right) ; E^{\prime}$ includes all edges of such a complete graph other than those in $E$. Moreover, for each $G_{i} \in \mathcal{G}-\mathcal{G}^{\prime}$, any $G_{i}^{k}$ has some edge in $E^{\prime}$ incident to it; $\bigcup_{G_{i} \in \mathcal{G}-\mathcal{G}^{\prime}} \bigcup_{k} V\left(G_{i}^{k}\right)$ has at least $\left\lceil\sum_{G_{i} \in \mathcal{G}-\mathcal{G}^{\prime}} g_{i} / 2\right\rceil$ edges in $E^{\prime}$ incident to it. Thus, intuitively, $E^{\prime}$ contains the edges connecting any two corresponding centers $c_{i}$ for some family $\mathcal{G}^{\prime}$ of $G_{i}$ and at least $\left\lceil\sum_{G_{i} \in \mathcal{G}-\mathcal{G}^{\prime}} g_{i} / 2\right\rceil$ edges incident to some $G_{i}^{k}$ with $G_{i} \in \mathcal{G}-\mathcal{G}^{\prime}$. Let

$$
\begin{aligned}
& f_{1}(i, j)=\left|\left\{\left(c_{i}, c_{j}\right)\right\}-E\right|+\left\lceil\frac{1}{2} \sum_{\ell \in\{1,2, \ldots, t\}-\{i, j\}} g_{\ell}\right\rceil, 1 \leq i<j \leq t, \\
& f_{2}(i, j, k)=\left|\left\{\left(c_{i}, c_{j}\right),\left(c_{j}, c_{k}\right),\left(c_{k}, c_{i}\right)\right\}-E\right|+\left\lceil\frac{1}{2} \sum_{\ell \in\{1,2, \ldots, t\}-\{i, j, k\}} g_{\ell}\right\rceil, \\
& 1 \leq i<j<k \leq t \\
& f_{3}(r)=\frac{r^{2}}{2}-\frac{3 r}{2}+1+\left\lceil\frac{1}{2} \sum_{r+1 \leq \ell \leq t} g_{\ell}\right\rceil, 1 \leq r \leq t .
\end{aligned}
$$

Note that in the case of $\mathcal{G}^{\prime}=\left\{G_{i}, G_{j}\right\}$ (resp., $\mathcal{G}^{\prime}=\left\{G_{i}, G_{j}, G_{k}\right\}$, resp., $\mathcal{G}^{\prime}=\left\{G_{1}, G_{2}, \ldots, G_{r}\right\}$ ), the first term of $f_{1}(i, j)$ (resp., $f_{2}(i, j, k)$, resp., $f_{3}(r)$ ) indicates the number of edges connecting $c_{i}, c_{j}$ (resp., any two vertices in $\left\{c_{i}, c_{j}, c_{k}\right\}$, resp., any two vertices in $\left.\left\{c_{1}, c_{2}, \ldots, c_{r}\right\}\right)$ to be included in $E^{\prime}$ and its second term indicates the number of edges incident to $\bigcup_{G_{i} \in \mathcal{G}-\mathcal{G}^{\prime}} V\left(G_{i}\right)$ to be included in $E^{\prime}$. We can prove that $O P T_{A}(G, 2 R+1) \geq \min \left\{\min _{1 \leq i<j \leq t} f_{1}(i, j)\right.$, $\left.\min _{1 \leq i<j<k \leq t} f_{2}(i, j, k), \min _{r \in\{1,2, \ldots, t\}-\{2,3\}} f_{3}(r)\right\}$.

Lemma $13 O_{P P} T_{A}(G, 2 R+1) \geq f(G)$ holds, where

$$
f(G)=\min \left\{\min _{1 \leq i<j \leq t} f_{1}(i, j), \min _{1 \leq i<j<k \leq t} f_{2}(i, j, k), \min _{r \in\{1,2, \ldots, t\}-\{2,3\}} f_{3}(r)\right\} .
$$

PROOF. Let $E^{\prime}$ be an arbitrary feasible solution to APD with a forest $G$ and $D=2 R+1$. Let $\mathcal{G}^{\prime} \subseteq \mathcal{G}$ be the family of subgraphs $G_{i}$ such that some $G_{i}^{j}$ satisfies $V\left(G_{i}^{j}\right) \cap V\left[E^{\prime}\right]=\emptyset$. 
Claim $14 E \cup E^{\prime}$ contains the edge $\left(c_{i}, c_{j}\right)$ for every pair of graphs $G_{i}, G_{j} \in \mathcal{G}^{\prime}$.

PROOF. Let $v_{i} \in V\left(G_{i}^{h}\right)$ and $v_{j} \in V\left(G_{j}^{k}\right)$ be two distant leaves with $V\left(G_{i}^{h}\right) \cap$ $V\left[E^{\prime}\right]=\emptyset=V\left(G_{j}^{k}\right) \cap V\left[E^{\prime}\right]$, and $H^{\prime}=\left(V, E \cup E^{\prime}\right)$. Then the edge $\left(c_{i}, c_{j}\right)$ must be included in $E \cup E^{\prime}$, since otherwise Lemma 12 implies that $d_{H^{\prime}}\left(v_{i}, v_{j}\right)>$ $2 R+1$ would hold, contradicting the feasibility of $E^{\prime}$.

This claim implies that the set $\left\{c_{i} \mid G_{i} \in \mathcal{G}^{\prime}\right\}$ of vertices induces a complete graph in $H^{\prime}$; the edge set $E_{1}^{\prime}=\left\{\left(c_{i}, c_{j}\right) \notin E \mid\left\{G_{i}, G_{j}\right\} \subseteq \mathcal{G}^{\prime}\right\} \subseteq E^{\prime}$ holds.

Moreover, every $G_{i}^{j}$ in $G_{i} \in \mathcal{G}-\mathcal{G}^{\prime}$ has some edge in $E^{\prime}$ incident to $V\left(G_{i}^{j}\right)$. Let $E_{2}^{\prime}$ be the set of all edges in $E^{\prime}$ incident to some vertex in $\bigcup_{G_{i} \in \mathcal{G}-\mathcal{G}^{\prime}} \bigcup_{j} V\left(G_{i}^{j}\right)$. Since one edge can contribute to two distinct $V\left(G_{i}^{j}\right)$ and $V\left(G_{k}^{\ell}\right)$, we have $\left|E_{2}^{\prime}\right| \geq\left\lceil\sum_{G_{i} \in \mathcal{G}-\mathcal{G}^{\prime}} g_{i} / 2\right\rceil$. Note that $E_{1}^{\prime} \cap E_{2}^{\prime}=\emptyset$ holds since $E_{1}^{\prime}$ consists of edges connecting two distinct vertices in $\left\{c_{i} \mid G_{i} \in \mathcal{G}\right\}$ and each edge in $E_{2}^{\prime}$ is incident to some vertex in $V-\left\{c_{i} \mid G_{i} \in \mathcal{G}\right\}$. Hence we have $\left|E^{\prime}\right| \geq$ $\left|E_{1}^{\prime}\right|+\left|E_{2}^{\prime}\right|$. Let $f^{\prime}\left(\mathcal{G}^{\prime}\right)=\left|E_{1}^{\prime}\right|+\left|E_{2}^{\prime}\right|$ and $f^{\prime \prime}(r)=\min \left\{f^{\prime}\left(\mathcal{G}^{\prime}\right)|| \mathcal{G}^{\prime} \mid=r\right\}$. From the definition of $f_{1}$ and $f_{2}$, we have $f^{\prime \prime}(2)=\min _{1 \leq i<j \leq t} f_{1}(i, j)$ and $f^{\prime \prime}(3)=$ $\min _{1 \leq i<j<k \leq t} f_{2}(i, j, k)$.

Assume that $r=\left|\mathcal{G}^{\prime}\right| \geq 1$ holds. Since $G$ is a forest, the graph induced by $\left\{c_{i} \mid G_{i} \in \mathcal{G}^{\prime}\right\}$ has at most $r-1$ edges in $E$, which implies that we have $\left|E_{1}^{\prime}\right| \geq r(r-1) / 2-(r-1)=(r-1)(r-2) / 2$. Moreover, we can see that $\left|E_{2}^{\prime}\right| \geq\left\lceil\sum_{i=r+1}^{t} g_{i} / 2\right\rceil$ holds since $g_{1} \geq g_{2} \geq \cdots \geq g_{t}$ holds. Thus, $f^{\prime}\left(\mathcal{G}^{\prime}\right) \geq$ $f^{\prime \prime}(r) \geq f_{3}(r)$ holds.

Now note that each $G_{i} \in \mathcal{G}$ satisfies $g_{i} \geq 1$. Hence, we can see that $f^{\prime \prime}(0) \geq$ $f^{\prime \prime}(1)$ holds, since $\left|E_{1}^{\prime}\right|=0$ satisfies in both cases of $\left|\mathcal{G}^{\prime}\right|=0$ and $\left|\mathcal{G}^{\prime}\right|=$ 1. Consequently, $\left|E^{\prime}\right| \geq \min _{0 \leq r \leq t} f^{\prime \prime}(r) \geq \min \left\{f^{\prime \prime}(2), f^{\prime \prime}(3), \min _{r \in\{1,2, \ldots, t\}-\{2,3\}}\right.$ $\left.f_{3}(r)\right\}$ holds, which proves the lemma.

The following lemma shows that we can find an edge set $E_{3}$ with $\left|E_{3}\right| \leq 2 f(G)$ and $d_{\left(V, E \cup E_{3}\right)}(u, v) \leq 2 R+1$ for every two distant leaves $u$ and $v$ (see Fig. 2(ii)).

Lemma 15 For an edge set $E_{3}$ chosen according to the following conditions (i)-(iii), we have $\left|E_{3}\right| \leq 2 f(G)$ and $d_{\left(V, E \cup E_{3}\right)}(u, v) \leq 2 R+1$ for every two distant leaves $u$ and $v$.

(i) If $f(G)=f_{1}(i, j)$ holds for some $1 \leq i<j \leq t$, then let $E_{3}=\left\{\left(c_{i}, c_{j}\right)-E\right\}$ $\cup\left\{\left(c_{i}, a_{\ell}^{h}\right) \mid i \neq \ell \neq j, 1 \leq h \leq g_{\ell}\right\}$.

(ii) If $f(G)=f_{2}(i, j, k)$ holds for some $1 \leq i<j<k \leq t$, then let $E_{3}=$ $\left(\left\{\left(c_{i}, c_{j}\right),\left(c_{j}, c_{k}\right),\left(c_{k}, c_{i}\right)\right\}-E\right) \cup\left\{\left(c_{i}, a_{\ell}^{h}\right) \mid \ell \notin\{i, j, k\}, 1 \leq h \leq g_{\ell}\right\}$. 

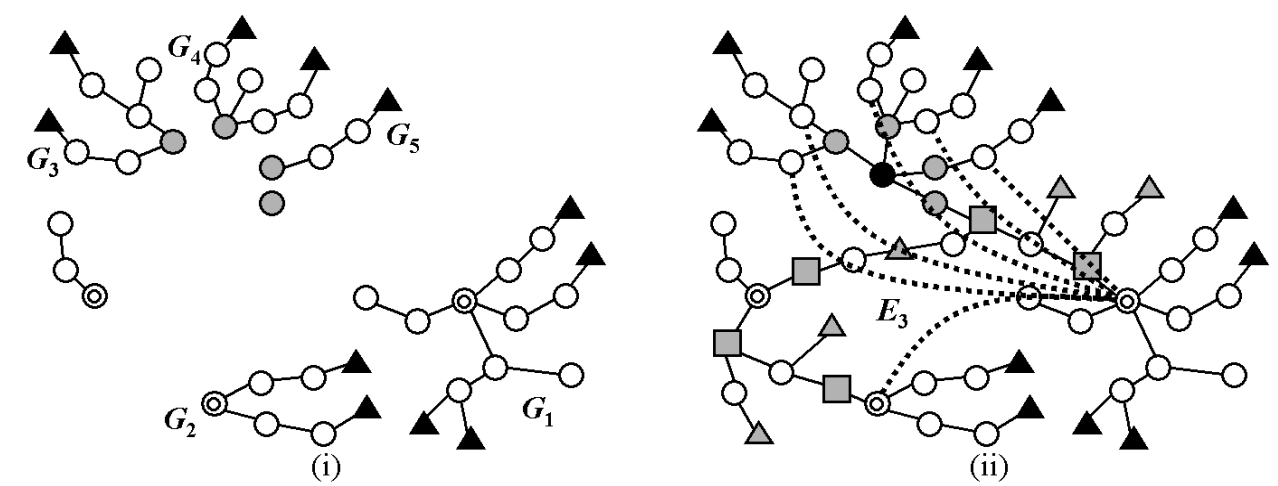

Fig. 2. (i) Illustration of components in $G^{\prime}$ having a center in $C_{1} \cup C_{2}$, and $\mathcal{G}=\left\{G_{1}, G_{2}, \ldots, G_{5}\right\}$, which are obtained from $G$ in Fig. 1 , where $g_{1}=3$, $g_{2}=g_{3}=g_{4}=2$, and $g_{5}=1$ hold. (ii) Illustration of a set $E_{3}$ of broken edges based on $f(G)$, where $f(G)=f_{1}(1,2)=4$ holds.

(iii) If $f(G)=f_{3}(r)$ holds for $r=1$ or some $4 \leq r \leq t$, then let $E_{3}=$ $\left\{\left(c_{i}, c_{j}\right) \notin E \mid 1 \leq i<j \leq r\right\} \cup\left\{\left(c_{1}, a_{\ell}^{h}\right) \mid r+1 \leq \ell \leq t, 1 \leq h \leq g_{\ell}\right\}$.

PROOF. First we show $\left|E_{3}\right| \leq 2 f(G)$. The cases of (i) and (ii) are clear from the definition of $f_{1}$ and $f_{2}$, respectively. The case of (iii) follows since $\left|\left\{\left(c_{i}, c_{j}\right) \notin E \mid 1 \leq i<j \leq r\right\}\right| \leq r(r-1) / 2 \leq 2\left(r^{2} / 2-3 r / 2+1\right)$ holds by $r=1$ or $r \geq 4$.

Let $C^{\prime}=\left\{c_{i}, c_{j}\right\}$ and $c^{\prime}=c_{i}$ (resp., $C^{\prime}=\left\{c_{i}, c_{j}, c_{k}\right\}$ and $c^{\prime}=c_{i}$, resp., $C^{\prime}=\left\{c_{1}, \ldots, c_{r}\right\}$ and $c^{\prime}=c_{1}$ ) in the case of (i) (resp., (ii), resp., (iii)). Let $H_{3}^{\prime}=\left(V, E \cup E_{3}\right)$. Then for each distant leaf $v \in V\left(G_{h}^{\ell}\right)$, we have $d_{H_{3}^{\prime}}\left(c^{\prime}, v\right) \leq$ $d_{H_{3}^{\prime}}\left(a_{h}^{\ell}, v\right)+1=R$ if $c_{h} \notin C^{\prime}$ holds, and we have $d_{H_{3}^{\prime}}\left(c^{\prime}, v\right) \leq R+1$ if $c_{h} \in C^{\prime}$ holds. Moreover, for every two distant leaves $v^{\prime} \in V\left(G_{h}\right)$ and $v^{\prime \prime} \in V\left(G_{k}\right)$ with $\left\{c_{h}, c_{k}\right\} \subseteq C^{\prime}$, we have $d_{H_{3}^{\prime}}\left(v^{\prime}, v^{\prime \prime}\right) \leq 2 R+1$ by $\left(c_{h}, c_{k}\right) \in E_{3} \cup E$. Therefore we see that $d_{H_{3}^{\prime}}(u, v) \leq 2 R+1$ holds for every pair of distant leaves $u$ and $v$.

The procedure of Step 3 is described as follows.

Step 3: Let $G^{\prime}$ be the forest obtained from $G$ by removing the set $C_{3} \cup\left\{c^{*}\right\}$ of vertices and the set $\left\{\left(c, c^{\prime}\right) \in E \mid c, c^{\prime} \in C_{1} \cup C_{2}\right\}$ of edges. Let $G_{i}, i=1,2, \ldots, t$ be the component containing $c_{i} \in C_{1} \cup C_{2}$ and $N_{G_{i}}^{R}\left(c_{i}\right) \neq \emptyset$. Compute a lower bound $f(G)$ on $\operatorname{OPT}_{A}(G, D)$ based on $\mathcal{G}=\left\{G_{1}, \ldots, G_{t}\right\}$. Let $E_{3}$ be the set of edges as defined in Lemma 15. Halt after outputting $E^{*}=E_{1} \cup E_{2} \cup E_{3}$.

Lemma 16 Algorithm $O D D-A P D(G, D)$ can be implemented to run in $O\left(n^{3}\right)$ time.

PROOF. An edge set $E_{1}$ can be found in $O\left(n^{2}\right)$ time by Theorem 6 . In 
Step 2, it is not difficult to see that a vertex set $C_{3}$ can be found in linear time by the depth first search [16] from $c^{\ell}$ in each $G^{\ell}$. So $E_{2}$ can be found in $O(n)$ time. In Step 3, computing $f(G)$ takes $O\left(n^{3}\right)$ time. In total, algorithm $\operatorname{ODD}-\operatorname{APD}(G, D)$ can be implemented to run in $O\left(n^{3}\right)$ time.

Summarizing the argument given so far, Theorem 4 is now established.

\section{BAPD with a forest}

In this section, we propose an algorithm, named $\operatorname{ODD}-\operatorname{BAPD}(G, D)$, which delivers a $(4,2)$-approximate (resp., (6,3)-approximate) solution to BAPD with a forest $G$ and an odd $D$ in $O(n)$ time if $D \geq 5$ (resp., $D=3$ ) holds (recall the definition of $(a, b)$-approximations in Definition 1).

Let $G=(V, E)$ be a forest with $|V| \geq 3$ and $I(G)$ be the set of isolated vertices $v \in V$ with $N_{G}(v)=\emptyset$. The algorithm $\operatorname{ODD}-\operatorname{BAPD}(G, D)$ consists of three steps. In the first step, we compute a set $C_{1}$ of centers such that $L(G) \subseteq C_{1}$ holds, a family of $R$-balls with centers in $C_{1}$ covers $V$, and the number $\left|C_{1}\right|$ of centers is minimized (recall the definition of $L(G)$ given in Section 2). Pick up two centers $c_{1}, c_{2} \in C_{1}$ and let $E_{1}=\left\{\left(c_{1}, c_{2}\right)\right\} \cup\left\{\left(c_{1}, c\right) \mid\right.$ $\left.c \in C_{1}-I(G)-\left\{c_{1}, c_{2}\right\}\right\} \cup\left\{\left\{\left(c_{1}, c\right),\left(c_{2}, c\right)\right\} \mid c \in I(G)-\left\{c_{1}, c_{2}\right\}\right\}$ (note that $\left|C_{1}\right| \geq|L(G)| \geq 2$ holds, because a forest $G$ with $|V| \geq 3$ satisfies $|L(G)| \geq 2$ ). Note that for the biconnectivity, each leaf (resp., isolated vertex) in $G$ must be incident to at least one added edge (resp., at least two added edges). Since each leaf in $G$ is incident to $E_{1}$, no distant vertex is a leaf in $G$. In the second step, we add a set $E_{2}$ of new edges with $\left|E_{2}\right| \leq\left|C_{1}\right|$ for $D \geq 5$ (resp., $\left|E_{2}\right| \leq 2\left|C_{1}\right|$ for $D=3$ ) in order to reduce the distance between every two distant vertices to at most $D$, by a slightly modified procedure from Step 2 in algorithm ODD$\operatorname{APD}(G, D)$. In the last step, we replace some edges in $E_{1} \cup E_{2}$ in order to attain the biconnectivity. More precise description of the algorithm is given as follows.

\section{Algorithm ODD-BAPD $(G, D)$}

Input: A forest $G=(V, E)$ and an odd integer $D \geq 3$.

Output: An edge set $E^{*}$ with $\operatorname{diam}\left(\left(V, E \cup E^{*}\right)\right) \leq D, \kappa\left(\left(V, E \cup E^{*}\right)\right) \geq 2$, and $\left|E^{*}\right| \leq 4 O P T_{A}(G, D)+2$ for $D \geq 5$ (resp., $\left|E^{*}\right| \leq 6 O P T_{A}(G, D)+3$ for $D=3)$.

Step 1: Let $R=\frac{D-1}{2}$. Compute a set $C_{1}$ of centers with the minimum cardinality such that $L(G) \subseteq C_{1}$ holds and the family of $R$-balls with centers in 
$C_{1}$ covers $V$. Choose two distinct vertices $c_{1}, c_{2} \in C_{1}$. Let $E_{1}=\left\{\left(c_{1}, c_{2}\right)\right\} \cup$ $\left\{\left(c_{1}, c\right) \mid c \in C_{1}-I(G)-\left\{c_{1}, c_{2}\right\}\right\} \cup\left\{\left\{\left(c_{1}, c\right),\left(c_{2}, c\right)\right\} \mid c \in I(G)-\left\{c_{1}, c_{2}\right\}\right\}$.

Step 2: Regard each component $G^{\ell}$ of $G$ as a rooted tree by choosing its root $c^{\ell}$ as $c_{1}$ if $c_{1} \in V\left(G^{\ell}\right)$ and as an arbitrary vertex in $C_{1} \cap V\left(G^{\ell}\right)$ otherwise. For each vertex $c_{i} \in C_{1}$, let $p\left(c_{i}\right) \in C_{1}$ be the nearest ancestor of $c_{i}$ in $G^{\ell}$ and let $a_{i}, b_{i}$ be vertices in $V\left(P_{c_{i}, p\left(c_{i}\right)}\right)$ with $d_{G}\left(c_{i}, a_{i}\right)=R$ and $d_{G}\left(c_{i}, b_{i}\right)=R+1$ if $d_{G}\left(c_{i}, p\left(c_{i}\right)\right) \geq R+1$ holds, and $a_{i}=b_{i}=\emptyset$ otherwise. Let $Q_{i}=\left\{a_{i}\right\}$ (resp., $\left.Q_{i}=\left\{a_{i}, b_{i}\right\}\right)$ for each vertex $c_{i} \in C_{1}-\left\{c_{1}\right\}$ if $R \geq 2$ (resp., $R=1$ ) holds. Let $E_{2}=\left\{\left(c_{1}, v\right) \mid v \in \bigcup_{c_{i} \in C_{1}-\left\{c_{1}\right\}} Q_{i}\right\}$.

Step 3: For each component $G_{i}^{\prime}$ of $G$ with $\left\{c_{1}, c_{2}\right\} \cap V\left(G_{i}^{\prime}\right)=\emptyset$ and $\left|V\left(G_{i}^{\prime}\right)\right| \geq 2$, pick up arbitrarily one edge $\left(c, c_{1}\right) \in E_{1}$ with $c \in V\left(G_{i}^{\prime}\right)$ and replace the edge $\left(c, c_{1}\right)$ with a new edge $\left(c, c_{2}\right)$. In the component $G^{\prime}$ of $G$ with $c_{1} \in V\left(G^{\prime}\right)$, for the set $L_{1}$ of leaves $v \in L\left(G^{\prime}\right)-\left\{c_{1}\right\}$ with $c_{2} \notin V\left(P_{v, c_{1}}\right)$, replace each edge $\left(c_{1}, v\right) \in E_{1}$ with $v \in L_{1}$ with a new edge $\left(c_{2}, v\right)$. Let $E_{1}^{\prime}$ be the edge set obtained from $E_{1}$ by this procedure. Halt after outputting $E^{*}=E_{1}^{\prime} \cup E_{2}$.

We prove the correctness of algorithm $\operatorname{ODD}-\operatorname{BAPD}(G, D)$ by the following lemmas. The next lemma shows $\left|E_{1}\right| \leq\left|C_{1}\right|+|I(G)|-1 \leq 2 O P T_{B}(G, 2 R+$ $1)+1$.

Lemma $17\left|C_{1}\right|+|I(G)| \leq 2 O P T_{B}(G, 2 R+1)+2$ holds.

PROOF. Let $E^{*}$ be an optimal solution to BAPD with $G$ and $D=2 R+1$, $W=V\left[E^{*}\right]$, and $H^{*}=\left(V, E \cup E^{*}\right)$. Since $\kappa\left(H^{*}\right) \geq 2$ holds, we have $L(G) \subseteq W$ and at least two edges in $E^{*}$ are incident to each $v \in I(G)$. Thus, $\left|E^{*}\right| \geq$ $(|W|+|I(G)|) / 2$ holds.

Now we consider the family $\mathcal{B}^{*}$ of $R$-balls with centers in $C_{1}$ in $G$. Let $X=$ $V-\left(\bigcup_{B \in \mathcal{B}^{*}} B\right) ; X$ is the set of vertices which are not contained in any $R$-ball $B \in \mathcal{B}^{*}$. The set $X$ satisfies the following property.

Claim 18 Every two vertices $x, y \in X$ satisfy $d_{G}(x, y) \leq 2 R+1$.

PROOF. Assume by contradiction that some two vertices $x, y \in X$ satisfy $d_{G}(x, y)>2 R+1$. Since $E^{*}$ is feasible to BAPD, we have $d_{H^{*}}(x, y) \leq 2 R+1$. Hence, any path $P_{x, y}^{*}$ between $x$ and $y$ in $H^{*}$ with $\left|P_{x, y}^{*}\right| \leq 2 R+1$ contains at least one edge in $E^{*}$. For $P_{x, y}^{*}$, let $v_{x} \in W \cap V\left(P_{x, y}^{*}\right)$ and $v_{y} \in W \cap V\left(P_{x, y}^{*}\right)$ denote the vertex in $W \cap V\left(P_{x, y}^{*}\right)$ nearest to $x$ and $y$, respectively. Since neither $x$ nor $y$ is covered by $\mathcal{B}^{*}$, we have $d_{G}\left(x, v_{x}\right) \geq R+1$ and $d_{G}\left(y, v_{y}\right) \geq R+1$. Thus we have $\left|P_{x, y}^{*}\right| \geq 2 R+2$, a contradiction. 
By this claim and the properties that $G$ is a forest and the path between each pair of vertices is unique, we can see that the set $X$ is covered by the family of two $R$-balls with two centers $a$ and $b$ for some edge $(a, b) \in E$. Therefore, $\mathcal{B}^{*} \cup\left\{B_{G}(a, R), B_{G}(b, R)\right\}$ covers $V$, and we have $|W|+2 \geq\left|C_{1}\right|$ from the minimality of $\left|C_{1}\right|$. Hence, we have $\left|E^{*}\right| \geq(|W|+|I(G)|) / 2 \geq\left(\left|C_{1}\right|+|I(G)|-\right.$ 2)/2, from which $\left|C_{1}\right|+|I(G)| \leq 2 O P T_{B}(G, 2 R+1)+2$ holds.

From the construction of $C_{1}, H_{1}=\left(V, E \cup E_{1}\right)$ satisfies $d_{H_{1}}\left(c_{1}, v\right) \leq R+1$ for every vertex $v \in V$. Hence any distant vertex $v \in V$ satisfies $d_{H_{1}}\left(v, c_{1}\right)=R+1$. Moreover, by $L(G) \subseteq C_{1}$, any distant vertex $v \in V$ satisfies $v \notin L(G)$.

Lemma 19 For each distant vertex $v \in V$, we have $d_{H_{1}}\left(v, c_{1}\right)=R+1$ and there exists a path $P_{c_{h}, p\left(c_{h}\right)}$ in $G$ for some vertex $c_{h} \in C_{1}-\left\{c_{1}\right\}$ with $v \in$ $V\left(P_{c_{h}, p\left(c_{h}\right)}\right)$.

PROOF. The first statement was proven above. Let $v$ be a distant vertex. By $v \notin L(G)$ and $L(G) \subseteq C_{1}$, there is a path $P_{c_{i}, c_{j}}$ in $G$ for some vertices $c_{i}, c_{j} \in C_{1}$ with $v \in V\left(P_{c_{i}, c_{j}}\right)$. Thus, the second statement holds.

The following lemma shows that $\operatorname{diam}\left(H_{2}\right) \leq D$ holds for $H_{2}=\left(V, E \cup E_{1} \cup\right.$ $\left.E_{2}\right)$.

Lemma $20 H_{2}$ satisfies $d_{H_{2}}\left(c_{1}, v\right) \leq R$ for all vertices $v \in V$.

PROOF. Let $v \in V$ be a distant vertex. By Lemma $19, d_{G}\left(v, c_{i}\right)=R$ holds for some $c_{i} \in C_{1}-\left\{c_{1}\right\}$ and $d_{G}(v, c) \geq R$ holds for every $c \in C_{1}$. Again by Lemma 19, we can see that there exists a vertex $c_{h} \in C_{1}-\left\{c_{1}\right\}$ such that $v \in V\left(P_{c_{h}, p\left(c_{h}\right)}\right)$. If $c_{h}=c_{i}$ holds, then we have $d_{H_{2}}\left(v, c_{1}\right)=1 \leq R$ because $d_{G}\left(c_{h}, p\left(c_{h}\right)\right)=d_{G}\left(c_{h}, v\right)+d_{G}\left(v, p\left(c_{h}\right)\right) \geq 2 R \geq R+1$ implies $v=a_{h}$.

We consider the case of $p\left(c_{h}\right)=c_{i}$. Note that there exists a vertex $c_{k} \in C_{1}$ with $p\left(c_{k}\right)=p\left(c_{h}\right)=c_{i}$ and $R \leq d_{G}\left(c_{k}, v\right) \leq R+1$, since each vertex in $N_{G}(v)$ is contained in some ball with a center in $C_{1}$. Also note that $d_{G}\left(c_{k}, c_{i}\right) \geq R+1$ holds. The case of $d_{G}\left(c_{k}, v\right)=R$ indicates that $v=a_{k}$ holds by the above arguments, and hence the lemma is proven. Assume that $d_{G}\left(c_{k}, v\right)=R+1$ holds. If $R=1$ holds, then we have $v=b_{k}$, from which $d_{H_{2}}\left(v, c_{1}\right)=1 \leq R$ holds. If $R \geq 2$ holds, then the vertex $v^{\prime} \in V\left(P_{c_{k}, c_{i}}\right) \cap N_{G}(v)$ with $d_{G}\left(c_{k}, v^{\prime}\right)=R$ satisfies $v^{\prime}=a_{k}$, from which $d_{H_{2}}\left(v, c_{1}\right)=2 \leq R$ holds.

The following lemma proves the correctness of algorithm $\operatorname{ODD}-\operatorname{BAPD}(G, D)$. 
Lemma $21 A$ set $E_{1}^{\prime} \cup E_{2}$ of edges is feasible to BAPD with a forest $G$ and $D=2 R+1$ and satisfies $\left|E_{1}^{\prime} \cup E_{2}\right| \leq 4 O P T_{B}(G, D)+2$ (resp., $\left|E_{1}^{\prime} \cup E_{2}\right| \leq$ $6 O P T_{B}(G, D)+3$ ) if $R \geq 2$ (resp., $R=1$ ) holds.

PROOF. From the construction of $\left|E_{1}\right|$, we have $\left|E_{1}\right| \leq\left|C_{1}\right|-1+|I(G)|$. From the construction of $\left|E_{2}\right|$, we have $\left|E_{2}\right| \leq\left|C_{1}\right|-1$ if $R \geq 2$ holds, and $\left|E_{2}\right| \leq 2\left(\left|C_{1}\right|-1\right)$ if $R=1$ holds. By Lemma 17 and $\left|E_{1}\right|=\left|E_{1}^{\prime}\right|$, we have $\left|E_{1}^{\prime} \cup E_{2}\right| \leq 4 O P T_{B}(G, D)+2$ (resp., $6 O P T_{B}(G, D)+3$ ) if $R \geq 2$ (resp., $R=1$ ) holds.

Let $H_{2}^{\prime}=\left(V, E \cup E_{1}^{\prime} \cup E_{2}\right)$. By Lemma 20 and the construction of $E_{1}^{\prime}$, we can see that each vertex $v \in V$ satisfies $d_{H_{2}^{\prime}}\left(c_{1}, v\right) \leq R+1$ and every vertex $v \in V$ with $d_{H_{2}^{\prime}}\left(c_{1}, v\right)=R+1$ satisfies $d_{H_{2}^{\prime}}\left(c_{2}, v\right)=R$. Thus $d_{H_{2}^{\prime}}(u, v) \leq 2 R+1$ holds for every pair of vertices $u, v \in V$.

For proving that $\kappa\left(H_{2}^{\prime}\right) \geq 2$ holds, it suffices to show that each leaf in $L(G)$ belongs to a cycle containing the edge $\left(c_{1}, c_{2}\right)$ in $H_{2}^{\prime}$ (note that $\left(c_{1}, c_{2}\right) \in$ $E \cup E_{1}^{\prime} \cup E_{2}$ holds). The case of $L(G)-\left\{c_{1}, c_{2}\right\}=\emptyset$ is clear, since $G$ is a tree with only two leaves $c_{1}$ and $c_{2}$ by $|V| \geq 3$ and we have $\left(c_{1}, c_{2}\right) \in E \cup E_{1}^{\prime} \cup E_{2}$. Let $v \in L(G)-\left\{c_{1}, c_{2}\right\}$ be a leaf in $G$. The case of $v \in I(G)$ is also clear by $\left\{\left(v, c_{1}\right),\left(v, c_{2}\right)\right\} \subseteq E_{1}^{\prime}$.

Assume that $v \in V\left(G^{\prime}\right)$ holds for a component $G^{\prime}$ of $G$ with $\left|V\left(G^{\prime}\right)\right| \geq 2$ and $c_{1} \notin V\left(G^{\prime}\right)$. Let $u_{2} \in V\left(G^{\prime}\right) \cap C_{1}$ be a vertex with $\left(u_{2}, c_{2}\right) \in E_{1}^{\prime}$ if $c_{2} \notin V\left(G^{\prime}\right)$ holds (such $u_{2}$ exists from the construction of $E_{1}^{\prime}$ ), and $u_{2}=c_{2}$ if $c_{2} \in V\left(G^{\prime}\right)$ holds. Moreover, from $\left|L\left(G^{\prime}\right)\right| \geq 2$, there exists a vertex $u_{1} \in V\left(G^{\prime}\right) \cap C_{1}$ with $\left(u_{1}, c_{1}\right) \in E_{1}^{\prime}$. If $v \neq u_{2}$ (resp., $\left.v=u_{2}\right)$ holds, then the cycle $\left\{\left(v, c_{1}\right),\left(c_{1}, c_{2}\right)\right.$, $\left.\left(c_{2}, u_{2}\right)\right\} \cup E\left(P_{u_{2}, v}\right)$ (resp., $\left.\left\{\left(v, c_{2}\right),\left(c_{2}, c_{1}\right),\left(c_{1}, u_{1}\right)\right\} \cup E\left(P_{u_{1}, v}\right)\right)$ proves the claim, where let $\left(c_{2}, u_{2}\right)=\emptyset$ in the case of $c_{2}=u_{2}$.

Assume that $v \in V\left(G^{\prime}\right)$ holds for the component $G^{\prime}$ of $G$ with $\left|V\left(G^{\prime}\right)\right| \geq 2$ and $c_{1} \in V\left(G^{\prime}\right)$. If $v \in L_{1}$ holds, then the cycle $E\left(P_{v, c_{1}}\right) \cup\left\{\left(c_{1}, c_{2}\right),\left(c_{2}, v\right)\right\}$ proves the claim. If $v \notin L\left(G^{\prime}\right)-L_{1}$ holds, then the cycle $E\left(P_{v, c_{2}}\right) \cup\left\{\left(c_{2}, c_{1}\right),\left(c_{1}, v\right)\right\}$ proves the claim.

Lemma 22 Algorithm $O D D-B A P D(G, D)$ can be implemented to run in $O(n)$ time.

PROOF. By using the algorithm in [4], $C_{1}$ can be found in $O(n)$ time. In Step 2, it is not difficult to see that $\bigcup_{c_{i} \in C_{1}} Q_{i}$ can be computed in $O(n)$ time by the depth first search from the vertex $c^{\ell}$ in each $G^{\ell}$. In Step 3, $L_{1}$ is the set of leaves in components of $G^{\prime}-c_{1}$ not containing $c_{2}$, where $G^{\prime}$ denotes the component of $G$ with $c_{1} \in V\left(G^{\prime}\right)$. Hence $E_{1}^{\prime}$ can be computed in $O(n)$ time. 
Consequently, Lemmas 21 and 22 indicate that Theorem 5 holds.

Finally, we remark that Steps 1 and 2 in algorithm $\operatorname{ODD}-\operatorname{BAPD}(G, D)$ can find a set $E^{\prime}$ of edges such that we have $\operatorname{diam}\left(\left(V, E \cup E^{\prime}\right)\right) \leq D$ and $\left|E^{\prime}\right|$ is at most two over four times the optimal (resp., at most three over six times the optimal) in the case of $D \geq 5$ (resp., $D=3$ ), for any type of problems to which any feasible solution $E^{\prime}$ satisfies $L(G) \subseteq V\left[E^{\prime}\right]$. Since BAPD belongs to such a type of problems, we can prove that BAPD is approximable within some constant without computing $f(G)$ defined in (1). Now it is known in [17] (resp., [12]) that the problem of augmenting an initial graph up to $k$-edgeconnectivity (resp., $k$-vertex-connectivity) by adding the minimum number of new edges is polynomially solvable (resp., 2-approximable in polynomial time). Therefore, we see that the following property holds.

Corollary 23 For a forest $G$, APD with an additional requirement that the resulting augmented graph $G^{\prime}$ satisfies $\lambda\left(G^{\prime}\right) \geq k$ (resp., $\kappa\left(G^{\prime}\right) \geq k$ ) for $k \geq 2$, is $(5,2)$-approximable (resp., (6,2)-approximable) if $D$ is an odd $\geq 5$ and $(7,3)$ approximable (resp., $(8,3)$-approximable) if $D=3$ holds, where $\lambda(G)$ denotes the edge-connectivity of $G$.

\section{Conclusion}

We have shown that APD with a forest $G$ and an odd $D$ is approximable within a constant in polynomial time, by proposing an $O\left(n^{3}\right)$ time 8-approximation algorithm for the problem. For BAPD with a forest $G$ and an odd $D$, we have shown that a feasible solution $E^{\prime}$ with $\left|E^{\prime}\right| \leq 4 O P T_{B}(G, D)+2$ (resp., $\left.\left|E^{\prime}\right| \leq 6 O P T_{B}(G, 3)+3\right)$ can be found in $O(n)$ time if $D \geq 5$ (resp., $D=3$ ) holds. Both algorithms depend on the performance guarantee of Chepoi and Vaxes' algorithm [6] for APD with an even $D$. Hence, any better approximation algorithm for APD with an even $D$ improves the performance guarantee of approximating APD with an odd $D$. Actually, it is still open whether APD with a forest is NP-hard or not, and Chepoi and Vaxes [6] conjectured that a solution obtained by their algorithm for APD with a forest and an even $D$ is optimal when $\operatorname{OPT}_{A}(G, D)$ is sufficiently large.

It is also a future work to consider the problem for another class of graphs, while Chepoi and Vaxes' algorithm [6] works for a wider class of graphs containing forests as mentioned in Section 1.

\section{Acknowledgments}

We are grateful to the anonymous referee for careful reading and suggestions. This research was partially supported by the Scientific Grant-in-Aid from Min- 
istry of Education, Culture, Sports, Scienceand Technology of Japan.

\section{References}

[1] N. Alon, A. Gyárfás, M. Ruszinkó, Decreasing the diameter of bounded degree graphs, J. Graph Theory 35 (2000) 161-172.

[2] C. Berge, Hypergraphs, Elsevier, North-Holland, Amsterdam, 1989.

[3] A. Brandstädt, F. Dragan, V. Chepoi, V. Voloshin, Dually chordal graphs, SIAM J. Discrete Math. 11(3) (1998) 437-455.

[4] R. Chandrasekaran, A. Daughety, Location on tree networks: $p$-center and $n$ dispersion problems, Mathematics of Operations Research 6 (1) (1981) 50-57.

[5] V. Chepoi, (personal communication), 2003.

[6] V. Chepoi, Y. Vaxes, Augmenting trees to meet biconnectivity and diameter constraints, Algorithmica 33 (2002) 243-262.

[7] F. R. K. Chung, Diameter of graphs: old problems and new results, Congr Numer 60 (1987) 295-317.

[8] Y. Dodis, S. Khanna, Designing networks with bounded pairwise distance, Proc. 31st Annual ACM Symposium on Theory of Computing (1999) 750-759.

[9] P. Erdős, A. Gyárfás, M. Ruszinkó, How to decrease the diameter of trianglefree graphs, Combinatorica 18 (4) (1998) 493-501.

[10] K. P. Eswaran, R. E. Tarjan, Augmentation problems, SIAM J. Comput. 5 (1976) 653-665.

[11] A. Frank, Connectivity augmentation problems in network design, in Mathematical Programming: State of the Art 1994, J.R. Birge and K.G. Murty (Eds.), The University of Michigan, Ann Arbor, MI (1994) 34-63.

[12] B. Jackson, T. Jordán, A near optimal algorithm for vertex connectivity augmentation, Lecture Notes in Computer Science 1969, Algorithms and Computation (Proc. ISAAC '00) (2000) 312-325.

[13] C. Li, S. McCormick, D. Simchi-Levi, On the minimum-cardinality-boundeddiameter and the bounded-cardinality-minimum-diameter edge addition problems, Operations Research Letters 11 (1992) 303-308.

[14] H. Nagamochi, T. Ibaraki, Graph connectivity and its augmentation: applications of MA orderings, Discrete Applied Mathematics 123 (1) (2002) $447-472$.

[15] A. A. Schoone, H. L. Bodlaendar, J. van Leeuwen, Diameter increase caused by edge deletion, J. Graph Theory 11 (3) (1987) 409-427. 
[16] R. E. Tarjan, Depth-first search and linear graph algorithms, SIAM J. Comput. 1 (1972) 146-160.

[17] T. Watanabe, A. Nakamura, Edge-connectivity augmentation problems, J. Comput. System Sci. 35 (1987) 96-144. 